\title{
Subtypes of NanS-p sialate O-acetylesterase encoded by Stx2a bacteriophages ${ }^{\dagger}$
}

\author{
Stefanía B. Pascal ${ }^{1}$, Juan R. Lorenzo López ${ }^{1}$, Paula M. A. Lucchesi ${ }^{1}$ and Alejandra Krüger ${ }^{1, *}$ \\ 1 Centro de Investigación Veterinaria de Tandil (CIVETAN, CONICET-CIC-UNCPBA), Facultad de Ciencias \\ Veterinarias, Universidad Nacional del Centro de la Provincia de Buenos Aires, Tandil, Buenos Aires, \\ Argentina. \\ * Correspondence: akruger@vet.unicen.edu.ar. \\ + Presented at the 1st International Electronic Conference on Microbiology, 02-30 November 2020; Available \\ online: https://ecm2020.sciforum.net/ \\ Published: 02 November 2020
}

\begin{abstract}
Shiga toxin (Stx)-producing Escherichia coli strains are foodborne pathogens that can cause severe human diseases, such as haemorrhagic colitis and haemolytic-uraemic syndrome. Stxs are encoded by bacteriophages (Stx phages) which show remarkable variations in genome composition and harbour several genes of unknown function. Recently, a gene encoding a sialate O-acetylesterase (NanS-p) was identified in some relevant Stx2a phages and it was suggested that it could provide advantages for bacterial growth in the gut. The aim of this study was to analyse the presence and sequence of nanS-p genes in available Stx2a phage genomes. A total of 59 DNA sequences of Stx2a phages were extracted from the NCBI GenBank database with the BLAST program using the stxa sequence from phage $933 \mathrm{~W}$ as query sequence, either as complete phage genomes (45) or from bacterial genomes by subsequent analysis with PHASTER web server (14). Comparative analysis revealed that nanS-p was located downsteam stx $x_{2 a}$ in all genomes. Twenty different amino acid sequences of NanS-p were identified. Specifically, catalytic esterase domains showed only 11 possible sequences, with differences mainly observed in nine amino acid positions. Sequences corresponding to the N-terminal domain (DUF1737) showed three possible sequences, two of them closely related, while C-terminal domain was highly variable giving place to four groups with structural differences. Since sialate O-acetylesterase activity has been determined from particular Stx2a phages, new studies are necessary to evaluate if the NanS-p subtypes identified in the present study also differ in their biological activity.
\end{abstract}

Keywords: Stx2a bacteriophages; sialate O-acetylesterase; subtypes; sialic acids

\section{Introduction}

Shiga toxin-producing Escherichia coli (STEC) strains are zoonotic foodborne pathogens that can cause severe human diseases, including haemorrhagic colitis (HC) and haemolytic uraemic syndrome (HUS), a leading cause of acute renal failure in children [1,2]. The main sources of STEC infections are bovine meat, milk, dairy products, tap water, vegetables and fruit [3].

STEC strains produce Shiga toxins (Stx), which are one of the most potent bacterial toxins known [4]. There are 2 major types of Stx (Stx1 and Stx2) and several subtypes [5]. STEC strains harbouring the $s t x_{2 a}$ subtype have shown the highest rates of HUS, hospitalization and bloody diarrhea [3].

The genes encoding the Stx toxins are carried by bacteriophages integrated into the bacterial chromosome, and these bacteriophages (Stx phages) play an important role in the regulation of toxin production, the dissemination of $s t x$, and the emergence of new STEC strains [6-9]. Stx2 phages are called lambda-like because they carry several genes homologous with bacteriophage lambda. 
However, they also carry many genes of unknown function. Additionally, the members of Stx phage family are highly variable in genome composition [10-12].

Nübling et al. [13] described the function of a large open reading frame (ORF) encoded directly downstream the Stx2a operon in the reference prophage 933W from Escherichia coli O157:H7 strain EDL933. This Stx2a prophage-encoded protein was able to hydrolyse acetic acid from bovine submaxillary gland mucin and 5-N-acetyl-9-O-acetyl neuraminic acid (Neu5,9Ac2). This enzyme was designated NanS-p due to its homology to the bacterial NanS and the fact that it is prophage encoded [14]. Later studies demonstrated the function of NanS-p encoded by Stx prophages and other prophages from O157 EDL933 and O104 LB226692 strains in Neu5,9Ac2 utilization, suggesting that NanS-p could provide advantages for bacterial growth in the gut $[14,15]$.

The aim of this study was to perform a comprehensive analysis of the presence and sequence of nanS-p genes in sequenced Stx2a phages.

\section{Materials and Methods}

We searched the scientific literature for sequenced Stx2 phages and the GenBank DNA database for Stx2a sequences by using the BLASTN program (https://blast.ncbi.nlm.nih.gov/) with the stx2a sequence from the phage $933 \mathrm{~W}$ (X07865) as query sequence.

DNA sequences of Stx2a phages were retrieved from available complete phage genomes or from bacterial genomes by subsequent analysis with PHAge Search Tool Enhanced Release (PHASTER) web server (http://phaster.ca) [16]. The localization of nanS-p in the prophage genomes was determined by BLAST analysis of stx flanking regions, using the corresponding region of $933 \mathrm{~W}$ as query sequence, and by examination of the sequence annotations at GenBank.

The nanS-p ORFs were translated into their respective amino acid sequences using the Molecular Evolutionary Genetics Analysis (MEGA) software version 7 [17]. Conserved domains were recognized in the 933W NanS-p sequence (AAD25447.1) using the Conserved Domain Search Service (CD-Search) (https://www.ncbi.nlm.nih.gov/Structure/cdd/wrpsb.cgi) [18] and InterProScan (https://www.ebi.ac.uk/interpro/search/sequence/) [19] tools. Sequence alignments and phylogenetic trees were constructed using MEGA 7 software [17].

\section{Results}

A total of 59 DNA sequences of Stx2a phages/prophages (including 933W) were extracted from the GenBank database, either as complete phage genomes (45) or from bacterial genomes (14). Comparative analyses revealed that nanS-p was located downsteam stxa in all analysed phage genomes and identified the presence of 20 different amino acid sequences of NanS-p.

The regions corresponding to the two domains identified in 933W NanS-p (Figure 1) were used for a detailed analysis of the different NanS-p sequences. The sequences corresponding to the N-terminal domain (DUF1737, IPR013619/PF08410) showed less variation with three possible sequences, two of them closely related, while those matching the catalytic esterase domain (SASA, IPR005181/PF03629) were grouped in 11 sequences (Figure 2), with differences mainly observed in nine amino acid positions (Figure 1). The comparison of the C-terminal sequences showed high variability, giving place to four different groups with structural variations.

\section{NanS-p (645 aa)}

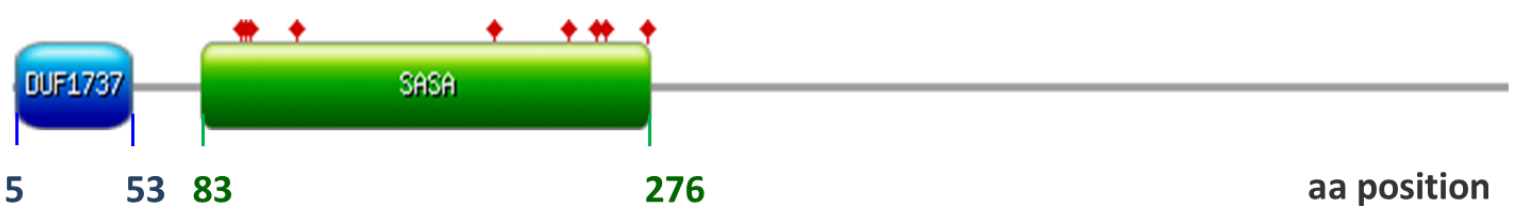

Figure 1. Graphical view of domains identified in NanS-p sequences. Numbers refer to the amino acid positions in the 933W NanS-p sequence (AAD25447.1). Red diamonds indicate the main variable 
positions in SASA domain. The scheme of the protein was generated with ExPASy PROSITE MyDomains Image Creator (http://prosite.expasy.org/mydomains/).

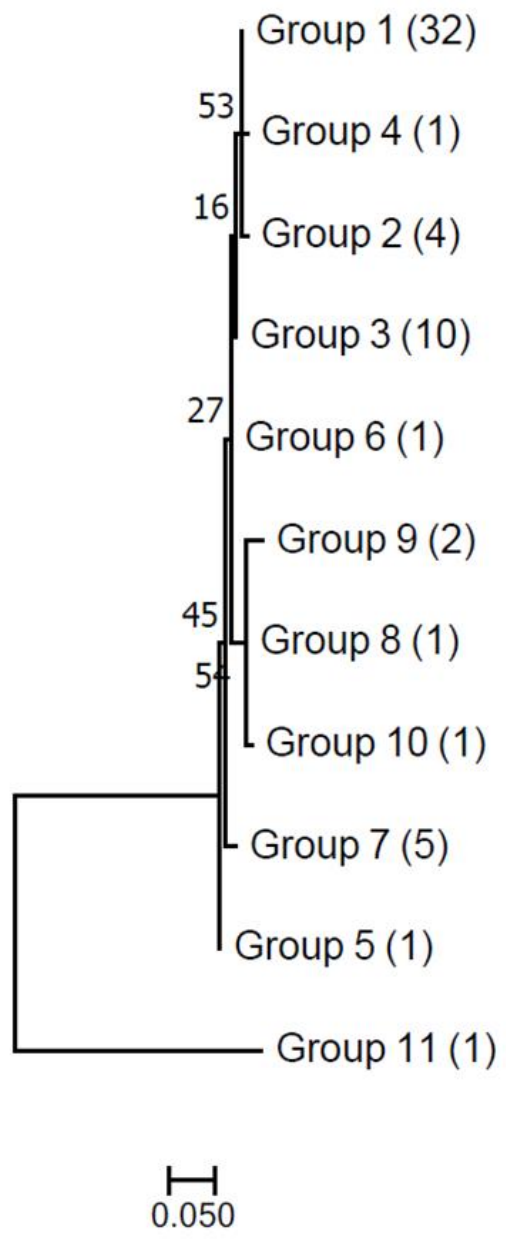

Figure 2. Phylogenetic tree of SASA domains of NanS-p proteins encoded by the 59 Stx phages included in the present study. Identical sequences were grouped and the molecular analysis involved 11 representative sequences. The evolutionary history was inferred by using the Maximum Likelihood method. Numbers in parentheses indicate the number of identical sequences in each group. Amino acid differences were concentrated at nine positions, except for one highly dissimilar sequence (group 11).

\section{Discussion}

Stx phages play key roles in STEC virulence because they not only carry Shiga toxin-encoding genes but also control their expression. However, little is known about the function of other phage encoded proteins and how they impact on the host $[12,20]$.

Previous studies have characterized NanS-p O-acetylesterases encoded by Stx2a-phages in O157 EDL933 and O104 LB226692 STEC strains that may contribute to bacterial growth in Neu5,9Ac2 rich environments, such as mucus [13-15]. Our study showed that NanS-p sialate O-acetylesterase was carried by all studied Stx2a phages suggesting an important role for Stx2a phage biology or for its bacterial host. Although its genome location was conserved, sequence differences were observed. Particularly, nine positions in the catalytic domain were shown to be variable. Further studies are necessary to evaluate if the NanS-p subtypes identified in the present study also differ in their biological activity.

\section{Conclusions}


Carriage of the nanS-p gene was observed in all the Stx2a phages analysed, being found at the same genomic location. The corresponding protein sequences showed a conserved domain organization, however, sequence differences were detected.

Author Contributions: conceptualization, P.L. and A.K.; methodology, S.P and J.L.; formal analysis, S.P., J.L. P.L. and A.K.; investigation, S.P.;-writing - original draft preparation, A.K.; writing - review and editing, S.P., J.L., P.L., A.K.; supervision, P.L and A.K.; funding acquisition, P.L and A.K.

Funding: This research was funded by FONCYT (Fondo para la Investigación Científica y Tecnológica), PICT 2018-03245 and CONICET (Consejo Nacional de Investigaciones Científicas y Técnicas), PIP 0662. S.P. holds a fellowship from CONICET.

Conflicts of Interest: The authors declare no conflict of interest. The funders had no role in the design of the study; in the collection, analyses, or interpretation of data; in the writing of the manuscript, or in the decision to publish the results.

\section{References}

1. Karmali, M.A. Infection by Shiga toxin-producing Escherichia coli: an overview. Mol. Biotechnol. 2004, 26, doi:10.1385/mb:26:2:117.

2. Karmali, M.A.; Petric, M.; Lim, C.; Fleming, P.C.; Arbus, G.S.; Lior, H. The association between idiopathic hemolytic uremic syndrome and infection by verotoxin-producing Escherichia coli. J. Infect. Dis. 1985, 151, 775-782, doi:10.1093/infdis/151.5.775.

3. Koutsoumanis, K.; Allende, A.; Alvarez-Ordóñez, A.; Bover-Cid, S.; Chemaly, M.; Davies, R.; De Cesare, A.; Herman, L.; Hilbert, F.; Lindqvist, R.; et al. Pathogenicity assessment of Shiga toxin-producing Escherichia coli (STEC) and the public health risk posed by contamination of food with STEC. EFSA J. 2020, 18, doi:10.2903/j.efsa.2020.5967.

4. Melton-Celsa, A.R. Shiga Toxin (Stx) Classification, Structure, and Function. Microbiol. Spectr. 2014, 2, doi:10.1128/microbiolspec.EHEC-0024-2013.

5. Scheutz, F.; Teel, L.D.; Beutin, L.; Pierard, D.; Buvens, G.; Karch, H.; Mellmann, A.; Caprioli, A.; Tozzoli, R.; Morabito, S.; et al. Multicenter evaluation of a sequence-based protocol for subtyping Shiga toxins and standardizing Stx nomenclature. J Clin Microbiol. 2012, 50, 2951-2963.

6. Wagner, P.L.; Acheson, D.W.K.; Waldor, M.K. Isogenic lysogens of diverse Shiga toxin 2-encoding bacteriophages produce markedly different amounts of Shiga toxin. Infect. Immun. 1999, 67.

7. Cornick, N.A.; Helgerson, A.F.; Mai, V.; Ritchie, J.M.; Acheson, D.W.K. In Vivo Transduction of an Stx-encoding phage in ruminants. Appl. Environ. Microbiol. 2006, 72, 5086-5088, doi:10.1128/AEM.00157-06.

8. Smith, D.L.; Wareing, B.M.; Fogg, P.C.M.; Riley, L.M.; Spencer, M.; Cox, M.J.; Saunders, J.R.; McCarthy, A.J.; Allison, H.E. Multilocus characterization scheme for Shiga toxin-encoding bacteriophages. Appl. Environ. Microbiol. 2007, 73, 8032-8040.

9. Beutin, L.; Hammerl, J.A.; Reetz, J.; Strauch, E. Shiga toxin-producing Escherichia coli strains from cattle as a source of the Stx2a bacteriophages present in enteroaggregative Escherichia coli O104:H4 strains. Int. J. Med. Microbiol. 2013, 303, 595-602, doi:http://dx.doi.org/10.1016/j.jimm.2013.08.001.

10. Smith, D.L.; Rooks, D.J.; Fogg, P.C.M.; Darby, A.C.; Thomson, N.R.; McCarthy, A.J.; Allison, H.E. Comparative genomics of Shiga toxin encoding bacteriophages. BMC Genomics 2012, 13, 311, doi:10.1186/1471-2164-13-311.

11. Steyert, S.R.; Sahl, J.W.; Fraser, C.M.; Teel, L.D.; Scheutz, F.; Rasko, D.A. Comparative genomics and stx phage characterization of LEE-negative Shiga toxin-producing Escherichia coli. Front. Cell. Infect. Microbiol. 2012, 2, 133, doi:10.3389/fcimb.2012.00133.

12. Mondal, S.I.; Islam, M.R.; Sawaguchi, A.; Asadulghani, M.; Ooka, T.; Gotoh, Y.; Kasahara, Y.; Ogura, Y.; Hayashi, T. Genes essential for the morphogenesis of the Shiga toxin 2-transducing phage from Escherichia coli O157:H7. Sci. Rep. 2016, 6, 39036, doi:10.1038/srep39036.

13. Nübling, S.; Eisele, T.; Stöber, H.; Funk, J.; Polzin, S.; Fischer, L.; Schmidt, H. Bacteriophage 933W encodes a functional esterase downstream of the Shiga toxin 2a operon. Int. J. Med. Microbiol. 2014, 304, 269-274, doi:http://dx.doi.org/10.1016/j.ijmm.2013.10.008.

14. Saile, N.; Voigt, A.; Kessler, S.; Stressler, T.; Klumpp, J.; Fischer, L.; Schmidt, H. Escherichia coli O157:H7 Strain EDL933 Harbors Multiple Functional Prophage-Associated Genes Necessary for the Utilization of 5N -Acetyl-9- O -Acetyl Neuraminic Acid as a Growth Substrate. Appl. Environ. Microbiol. 2016, 82, 5940- 
5950, doi:10.1128/AEM.01671-16.

15. Saile, N.; Schwarz, L.; Eißenberger, K.; Klumpp, J.; Fricke, F.W.; Schmidt, H. Growth advantage of Escherichia coli O104:H4 strains on 5- N -acetyl-9- O -acetyl neuraminic acid as a carbon source is dependent on heterogeneous phage-Borne nanS-p esterases. Int. J. Med. Microbiol. 2018, 308, 459-468, doi:10.1016/j.ijmm.2018.03.006.

16. Arndt, D.; Grant, J.R.; Marcu, A.; Sajed, T.; Pon, A.; Liang, Y.; Wishart, D.S. PHASTER: a better, faster version of the PHAST phage search tool. Nucleic Acids Res 2016, 44, W16-21, doi:10.1093/nar/gkw387.

17. Kumar, S.; Stecher, G.; Tamura, K. MEGA7: Molecular Evolutionary Genetics Analysis Version 7.0 for Bigger Datasets. Mol. Biol. Evol. 2016, 33, 1870-1874, doi:10.1093/molbev/msw054.

18. Marchler-Bauer, A.; Derbyshire, M.K.; Gonzales, N.R.; Lu, S.; Chitsaz, F.; Geer, L.Y.; Geer, R.C.; He, J.; Gwadz, M.; Hurwitz, D.I.; et al. CDD: NCBI's conserved domain database. Nucleic Acids Res 2015, 43, D222-6, doi:10.1093/nar/gku1221.

19. Jones, P.; Binns, D.; Chang, H.-Y.; Fraser, M.; Li, W.; McAnulla, C.; McWilliam, H.; Maslen, J.; Mitchell, A.; Nuka, G.; et al. InterProScan 5: genome-scale protein function classification. Bioinformatics 2014, 30, 12361240, doi:10.1093/bioinformatics/btu031.

20. Holt, G.S.; Lodge, J.K.; McCarthy, A.J.; Graham, A.K.; Young, G.; Bridge, S.H.; Brown, A.K.; Veses-Garcia, M.; Lanyon, C. V.; Sails, A.; et al. Shiga toxin encoding Bacteriophage $\phi 24 \mathrm{~B}$ modulates bacterial metabolism to raise antimicrobial tolerance. Sci. Rep. 2017, 7, 40424, doi:10.1038/srep40424.

Publisher's Note: MDPI stays neutral with regard to jurisdictional claims in published maps and institutional affiliations.

(C) 2020 by the authors. Submitted for possible open access publication under the terms and conditions of the Creative Commons Attribution (CC $\quad$ BY) license (http://creativecommons.org/licenses/by/4.0/). 